\title{
INVESTIGACIONES
}

\section{Un estudio comparado sobre la transición de Secundaria hacia la Universidad entre Brasil y España}

\author{
A comparative study on the transition from Secondary \\ to University between Brazil and Spain
}

\author{
Ariadne Runte Geidel ${ }^{a}$, Miguel Pérez Ferra ${ }^{a}$ \\ ${ }^{a}$ Departamento de Pedagogía de la Facultad de Humanidades y Ciencias de la Educación \\ de la Universidad de Jaén, España. \\ arunte@ujaen.es, mperez@ujaen.es
}

\begin{abstract}
RESUMEN
El objetivo de este estudio fue comparar la transición de Secundaria hacia la Universidad en estudiantes de carreras de Educación, entre una universidad brasileña y otra española. Se ha utilizado un instrumento ad hoc con 34 ítems que constituyeron cuatro factores, F1: Adaptación académica, F2: adaptación a la nueva vida, F3: adaptación a la Información, Horarios y Recursos, y F4: Autoestima y Autonomía. Los resultados encontrados muestran que los estudiantes de ambas universidades tienen la misma capacidad de adaptación a la vida académica, pero muestran diferencias significativas cuanto a la adaptación a la nueva vida y demás factores. Los estudiantes brasileños parecen adaptarse mejor a la nueva vida, informaciones y horarios, pero tienen menos autonomía y autoestima que el alumnado español.
\end{abstract}

Palabras claves: adaptación, educación superior, educación comparada.

\begin{abstract}
The objective of this study was to compare the transition from Secondary to University in students of careers in Education, between a Brazilian university and another Spanish university. We have used an ad hoc instrument with 34 items that constituted four factors, F1: Academic adaptation, F2: adaptation to the new life, F3: Adaptation to Information, Schedules and Resources and F4: Self-esteem and Autonomy. The results show that the students of both universities have the same ability to adapt to academic life, but show significant differences in terms of adaptation to the new life and other factors. Brazilian students seem to adapt better to new life, information and schedules, but they have less autonomy and self-esteem than Spanish students.
\end{abstract}

Key words: adaptation, superior education, comparative education. 


\section{INTRODUCCIÓN}

La idea de comparar la transición de la educación secundaria a la universitaria entre estudiantes del sur de Brasil (Porto Alegre) y del sur de España (Jaén) ha surgido pensando en dos contextos muy diferentes, a la vez que contrarios a lo esperado tradicionalmente, es decir, una universidad en Europa y otra en Sudamérica, pero la europea (Universidad de Jaén, UJA) frecuentada por una mayoría de estudiantes de ambiente rural y bajos niveles culturales familiares, en cuanto a la universidad sudamericana (Universidade Federal do Rio Grande do Sul, UFRGS), ubicada en la capital de uno de los Estados más prósperos de Brasil y, por lo tanto, frecuentada por una mayoría de estudiantes pertenecientes a un contexto urbano y familiar con buenos niveles culturales. El análisis comparativo de estas dos realidades ha permitido profundizar en el hecho de que sí determinados indicadores que dificultan la transición de los estudiantes de Secundaria a la Universidad se deben solo a carencias socioeconómicas o bien hay, en algunas ocasiones, otros aspectos influyentes.

La Universidad de Jaén fue creada en mil novecientos noventa y tres, siendo hasta esa fecha campus universitario de la Universidad de Granada. Realiza una gran labor social, ya que siendo esta provincia una de las de producto interior bruto (PIB) más bajo de España; posiblemente en función de esta peculiaridad, también se caracteriza por ser la de mayor porcentaje de primera generación universitaria de España, así, ha posibilitado el acceso a estudios superiores de un gran número de estudiantes que anteriormente no podían hacerlo. Económicamente, la provincia depende del monocultivo del olivar y algo de turismo rural, una realidad que genera una cultura que no fomenta el emprendimiento. Sin embargo, no se puede obviar que la formación de jóvenes universitarios, aunque no tenga unas consecuencias inmediatas en la prosperidad social, si presenta atisbos de cambios económico-culturales a medio plazo (Martín-Mesa y Alcalá-Olid, 2018).

La UFRGS es una universidad pública brasileña que inició su historia en 1895 con la fundación de algunas escuelas universitarias, pero como Universidad fue fundada oficialmente en 1934. Está localizada en la ciudad de Porto Alegre que es la capital del estado de Río Grande del Sur, el cual según el Jornal do Comercio (Rio Grande do Sul tiene la tercera mayor renta per cápita, 2018) es el quinto estado con mayor IDH (índice de desarrollo humano) de Brasil y, según el Instituto Brasileño de Geografía y Estadística (IBGE), tiene la tercera mayor renta per cápita del país, fundamentalmente en función de sus actividades económicas basadas tanto en la agricultura y ganadería, como en la industria, que al inicio del siglo XXI tuvo una expansión en las áreas de la petroquímica y de la tecnología de la información. Cerca de treinta mil personas estudian actualmente en la UFRGS por ser una de las instituciones más cualificadas de enseñanza superior en Brasil (CSCIC, s.f.).

La transición hacia la Universidad no ha sido muy estudiada en Brasil por lo que la literatura es escasa, cuestión mejor estudiada en España, razón por la que la literatura es más abundante (López-Cárdenas, Mella y Cáceres, 2019; Pérez-Ferra et al., 2018). Sin embargo, es un tema de interés, no solo científicamente hablando, sino también a nivel social, por las repercusiones sociales y económicas y, no menos importantes, de carácter afectivo-emocional, por la sensación de fracaso que pueden generar en los estudiantes.

Históricamente los estudios universitarios han sido enseñanzas a las que han tenido acceso solamente las élites de los países, y en eso España y Brasil no se diferencian. Con la progresiva democratización de los países y de sus políticas educativas, la Universidad se fue haciendo accesible a diferentes capas de población. Sin embargo, hay autores que 
defienden que la democratización se ha dado más en el acceso a la universidad que en el éxito académico (Almeida y Soares, 2004). Para estos autores, la "masificación" de la Universidad, no se ha dado solo en función del crecimiento exponencial del número de alumnos, sino que también está causada por el hecho de que la Universidad no ha ampliado recursos, instalaciones y sobre todo metodologías de enseñanza que atiendan a este nuevo contingente, que está formado no solo por una pequeña élite social.

La oferta de educación en Brasil ha crecido sustancialmente en los años noventa y dos mil en todos los niveles, pero aun así el porcentual de acceso a la Universidad era bastante bajo, cerca del 19\% (Andrade, 2012). Según datos más recientes el porcentual de jóvenes entre 18 y 24 años que estaban en la Universidad en 2014 llegó al 58,5\% (Ministerio de Educación de Brasil, 2017).

Es verdad que iniciar la vida universitaria es una transición compleja, pues pone a los estudiantes ante un nuevo contexto que presenta diferentes dificultades y retos, como separarse de la familia y amigos, superar las exigencias académicas o asumir nuevas responsabilidades, un conjunto de dificultades para las cuales algunos pueden no estar debidamente preparados y esto no está directamente relacionado con el nivel económico o cultural de las familias (Almeida y Soares, 2004). En Brasil, los problemas más frecuentes percibidos por los universitarios se refieren a la vida académica en sí, es decir los estudios, así como a aquellos vinculados con las relaciones interpersonales y de socialización, además de los problemas económicos (Pereira, 2004; Pachane, 2004). Almeida y Soares (2004), han identificado cuatro dimensiones en las que se agrupan estas nuevas dificultades: académica, social, personal y vocacional/institucional. Estas dimensiones se corresponderían a otras encontradas por Pérez-Ferra et al., 2018, que serían la "Integración del estudiante en la nueva realidad académico-social" (de ámbito social), "adaptación a los nuevos procesos didáctico-curriculares" (relativa a dimensión académica), "autonomía y seguridad personal" (de orden personal) y el "conocimiento y utilización de los servicios universitarios" (que podríamos relacionar con la vocacional/institucional).

Hay estudios que hacen hincapié sobre el estatus educativo y socio económico familiar, siendo el factor que mayormente influye sobre los resultados académicos de los estudiantes (Fajardo Bullón et al., 2017; Runte-Geidel y Femia, 2015; Gil Flores, 2011). Algunas de estas investigaciones han abordado si el nivel socioeconómico de los jóvenes influye en su capacidad de adaptación a la vida adulta, como es el caso del estudio de Dutra-Tomé y Koller (2014), realizado en la ciudad de Porto Alegre, el que encontró que los jóvenes con bajos niveles socioeconómicos tienden a asumir responsabilidades de la vida adulta de forma más precoz, pero que los jóvenes de clases sociales más altas presentan mayor indecisión en clasificarse como adultos o adolescentes.

Ciertamente, el nivel económico y cultural de las familias incide en una transición menos problemática de los estudiantes de Secundaria a la Universidad, pero no lo es menos, que hay otros condicionantes que no establecen diferencias significativas entre estudiantes que proceden de familias con elevados niveles culturales y económicos y otras en situaciones más desfavorecidas en estos aspectos. No se produce siempre correlación entre el rendimiento académico de los estudiantes y el nivel cultural de los padres en edades previas al ingreso en la universidad, situación determinada por el mayor nivel de independencia de los hijos en esa etapa, que hace que remita el seguimiento de los padres (Guevara, Jaramillo y Tovar, 2013; López-Betancourt, García-Rodríguez y DíasSoto, 2018). 
También la revisión de la bibliografía científica pone de manifiesto que la separación de los antiguos compañeros, muchos de ellos amigos, dificultad la relación con los nuevos, situación que dificulta el trabajo en equipo, generada por un desarraigo afectivo, que afecta a la autoestima y al autoconcepto, y cuyas consecuencias son notorios en los hábitos de estudio y en la seguridad a la hora de demostrar los logros académicos adquiridos o para abordar nuevas situaciones (Jansen \& Suhre, 2010).

La dimensión social y la capacidad del alumnado para integrase en nuevos grupos de compañeros y amigos, así como poder adaptarse a una nueva realidad cultural, depende también de sus condiciones personales. Uno de estos factores personales se refiere a que muchos jóvenes llegan a la Universidad con ideas equivocadas y una visión naíf sobre lo que se van a enfrentar y eso puede provocar, durante el primer año, una serie de desajustes emocionales, como el estrés o incluso algunas psicopatologías (Pereira y Cardoso, 2015). Un estudio de Bolsoni-Silva y Loureiro (2014) ha abordado las habilidades sociales desarrolladas por universitarios con ansiedad frente a universitarios sin este problema, y han encontrado que efectivamente, la ansiedad afecta habilidades como hablar en público u otras habilidades sociales. Parece evidente, tanto en Brasil como en España, que situaciones que debieran parecer habituales se tornan en "falta de planificación por parte de las instituciones (que) contribuye a que la transición educativa se convierta en inseguridad para el alumnado" (Álvarez-Teruel, Tortosa-Ybañez y Grau, 2012). Razón por la que la acción tutorial, el apoyo de estudiantes ya experimentados y la comunicación entre los orientadores de los centros y los vicerrectorados de estudiantes son vitales para paliar estos problemas.

La capacidad de adaptación académica, o "a los nuevos procesos didáctico curriculares" es influenciada tanto por las expectativas de los profesores que, siendo muy altas, no favorecen la integración del alumnado a la nueva situación, como por factores relacionados con las posibilidades de elección de la carrera que quieren cursar y otros relacionados específicamente con los conocimientos previos que traen de la enseñanza primaria y secundaria (Almeida y Soares, 2004). En ese sentido cobra relevancia el uso de metodología activa, centrada en la participación del alumnado para que se tornen responsables de sus aprendizajes, intercambien informaciones y desarrollen el pensamiento crítico y las actitudes colaborativas (Pérez-Ferra et al., 2018; Raya Díez, 2017; Díaz Barriga, 2013; Rodríguez Gallego, 2013). Dichos métodos, además, contribuyen a la motivación del alumnado para el aprendizaje. El estudio llevado a cabo por Marini y Boruchovitch (2014) sobre la autorregulación del aprendizaje en alumnos universitarios, entendiendo esta realidad como las estrategias utilizadas por el alumnado para planificar, dar seguimiento y reglar el propio aprendizaje, llegaron a la conclusión de que los universitarios con mayor motivación para aprender emplean mejores estrategias de autorregulación; es decir, las de tipo metacognitivo, a la vez que usan en menor medida las estrategias consideradas disfuncionales, tales como la ingesta de alcohol o drogas, o bien iniciar los procesos de estudio a última hora. Por el contrario, cuando los estudiantes asumen libremente una actividad sin ninguna recompensa asociada, sino por el hecho de que se orientan al aprendizaje, se genera una motivación que les permite su participación de manera voluntaria (Méndez-Giménez, Cecchini-Estrada y Fernández-Río, 2017; Vallerand, 2008).

No pocos investigadores han considerado la importancia de la motivación intrínseca, que es la que más incide en la autodeterminación. Esta motivación atiende a las razones para aprender, derivadas de la motivación inherente por el desarrollo de la misma actividad 
(Noels, 2001; Noels, Chaffee, Michalyk \& Sugita McEown, 2014). Esto es debido a que la motivación intrínseca representa la tendencia inherente a buscar desafíos y ejercitar las capacidades de los alumnos, razón por la que es esencial para el desarrollo cognitivo y social, que a su vez es fuente principal de bienestar (Deci \& Ryan, 1985; Jochen et al., 2016; Ryan \& Deci, 2000).

De todos modos, la adaptación de los estudiantes a los nuevos procesos didácticocurriculares, al ser el aprendizaje un proceso de reconstrucción del conocimiento, determinado por la interacción entre la experiencia personal del alumno y su contexto social, da lugar a que la realidad cultural incida positivamente en el aprendizaje, pero no es menos cierto que hay ciertas dificultades para comunicarse con el profesorado; para gestionar el tiempo de trabajo autónomo o para comprender la terminología de las materias, aspectos en los que no suele incidir tanto el nivel económico o cultural de las familias, sino la formación previa de los estudiantes, la capacidad de interacción del profesorado con los estudiantes, como el nivel de relación entre los centros de Secundarias y las universidades. Si bien, los procesos comunicativos son más ágiles cuando el nivel cultural es más elevado, lo que siendo importante no es determinante (Novo-Corti, MuñozCantero y Calvo-Porral, 2011).

Las capacidades de adaptación social y también académica se ven implicadas, a su vez, por los niveles de autorregulación de los aprendizajes y el grado de autonomía de los estudiantes. Algunos estudios detectaron bajos niveles en ambos aspectos en los estudiantes recen ingresados en la Universidad (Rosario et al., 2000; Valadas y Gonçalves, 2002). Por otro lado, se entiende la importancia de estos aspectos, tanto como la seguridad personal, para que este proceso tan complejo, como es la transición hacia la Universidad, se haga de forma positiva, pues permiten una adecuada toma de decisiones y asumir responsabilidades nuevas, ayudan a una mejor gestión del tiempo libre y el de estudios, además de facilitar el proceso participativo en clase (Nuñez, 2009).

Finalmente, el conocimiento y utilización de los servicios y recursos institucionales disponibles también son elementos importantes que influyen en la capacidad de una mejor o peor transición a la nueva etapa de vida. Las posibilidades de acceso a la información pertinente y necesaria y la adecuada utilización de los recursos disponibles dependen de lo que oferta la Universidad (Texeira, Castro y Piccolo, 2007). Es cierto que el

conocimiento y adecuada utilización de los servicios universitarios, agiliza los trámites y gestiones en la Universidad, aunque un desconocimiento de los procesos a seguir puede invertir la relación efectividad-tiempo utilizado. Los estudiantes no tienen excesiva dificultad para acceder a la información sobre diversos servicios del campus, el problema surge cuando han de interpretar la mencionada información (Brown, Wohn \& Ellison, 2016).

Lo que no resulta siempre fácil, fundamentalmente por dos razones; una porque el alumnado llega a la Universidad con carencias léxico-semánticas considerables; otra, porque el lenguaje administrativo, encierra procesos francamente crípticos, abundando la disociación real entre significantes y significados, que conducen en no pocas ocasiones, también entre el profesorado, a una ceremonia de la confusión. El usuario demanda la información de modo clarificador y desearía un acceso transparente e íntegro (García-Marco, 1995). Asimismo, hay otros componentes como pueden ser los servicios de apoyo (psicológico, psicopedagógico, 
etc.) que pueden favorecer o mejorar la capacidad de adaptación, facilitando la transición hacia la Universidad, algunos tienen un carácter paliativo y otros deben tener una perspectiva preventiva de intervención (Almeida y Soares, 2004).

Revisada la bibliografía se plantearían como objetivos de este estudio, conocer el nivel de integración de los estudiantes de primer año, respecto a su nueva realidad social y académica, así como su adaptación las nuevas prácticas didáctico-curriculares; identificar los niveles de autonomía y seguridad personal ante las nuevas situaciones que han de abordar, así como analizar el conocimiento y adecuada utilización que hacen los estudiantes de los servicios universitarios. Igualmente, se plantea identificar la existencia de diferencias estadísticamente significativas entre variables, como el país de residencia de los estudiantes, el sexto y la edad, sus calificaciones de acceso a la Universidad, así como si viven con la familia, o no durante el curso.

\section{METODOLOGÍA}

\subsection{PARTICIPANTES}

La población del estudio se constituye por los estudiantes de primer curso de grado en educación de las Universidades de Jaén y de la Universidade Federal do Rio Grande do Sul, en Brasil. El muestreo fue de conveniencia, es decir no aleatorio, justificándolo por su menor requerimiento desde los puntos de vista tanto económico como temporal. Este procedimiento permite obtener una alta tasa de participación dada la facilidad en la aplicación del instrumento (McMillan y Schumacher, 2001). La muestra está constituida por $\mathrm{N}=335$ estudiantes de los cuales el $85 \%$ son alumnos y alumnas de la Universidad de Jaén y el $15 \%$ restante lo son de la Universidad brasileña. En el conjunto total, cerca del $20 \%$ son hombres y un $80 \%$ son mujeres, con un $0,6 \%$ de participantes que eligieron una tercera opción en cuanto a su condición de género. La mayoría $(80 \%)$ tienen una edad comprendida entre los 17 y 21 años. Solo un 5\% es mayor de 26 años. Respecto a las calificaciones en las respectivas pruebas de acceso, el $41 \%$ ha sido de Aprobado, el $37 \%$ de Notable y el $21 \%$ de Sobresaliente. En cuanto al nivel educativo familiar, cerca de un 60\% tiene estudios básicos (Primaria y Secundaria), mientras que el 37\% tiene estudios universitarios con solo un $2,4 \%$ con estudios de posgrado.

\subsection{INSTRUMENTO}

El instrumento utilizado consta de 34 ítems con cinco opciones de respuesta que varían entre $1=$ "totalmente en desacuerdo" a 5= "totalmente de acuerdo". Previamente a la formulación de los ítems se presentan una serie de cuestiones relativas a los datos sociodemográficos: edad, sexo, nivel educativo familiar y cuestiones relacionadas con los estudios anteriores y la calificación en las pruebas de acceso, que constituyen las variables demográficas.

\subsubsection{Validez de Contenido}

Para estudiar la validez de contenido se ha considerado un juicio de expertos, consultando a profesores de la Facultad de Educación de la Universidade Federal do Rio Grande do 
Sul (Brasil) para la versión en portugués del instrumento. Se ha utilizado el IVC - Índice de Validez de Contenido de Lawshe (1975) para calcular la validez de cada ítem y del instrumento en general. Según este autor, si al menos un 50\% de los jueces consultados consideran un ítem del cuestionario como "esencial" eso significa que tal ítem tiene validez de contenido, es decir, si el cálculo del IVC alcanza valores positivos (entre 0 y 1 ) significa que la mitad o más de los jueces consultados consideraron el ítem válido para el instrumento. En este sentido, prácticamente todos los ítems del cuestionario (exceptuando 2 de ellos) fueron considerados válidos (IVC entre 0,33 y 1). El cálculo del IVC general del cuestionario ha alcanzado el valor de 0,6 poniendo de manifiesto que el mismo tiene buena validez de contenido.

\subsubsection{Análisis factorial y de fiabilidad}

Para detectar el número y dimensiones implícitas en el instrumento, se ha considerado un análisis factorial exploratorio (AFE) mediante componentes principales. Lo apropiado de dicha técnica de análisis sobre nuestra muestra se contrasta mediante el test de esfericidad de Batlett y la medida de adecuación muestral de Kaiser-Meyer-Olkin (KMO). Ambos procedimientos contrastan si las magnitudes de las correlaciones parciales entre los ítems del test son significativas. La adecuación viene dada por la significación del test de Bartlett $(P<5 \%)$ y por una medida KMO $>0,8$ (Hair et al., 2009). Tanto la significación del test de esfericidad de Barlett: $\mathrm{c}^{2}(561 \mathrm{gl})=6758, P<0,001$, como la medida de adecuación muestral de Kaiser-Meyer-Olkin $(\mathrm{KMO}=0,93>0,8)$, indican que la muestra considerada era susceptible de ser abordada mediante un análisis factorial.

Por otra parte, la fiabilidad o consistencia interna del instrumento, fue evaluada mediante el coeficiente alfa de Cronbach, cuyo límite inferior se acepta que sea un valor de 0,60 - 0,70 (Hair, Black, Babin \& Anderson, 2009). También se ha utilizado el coeficiente alfa de Cronbach sobre los ítems integrantes de cada uno de los factores obtenidos en el AFE. El coeficiente alfa de Cronbach presenta un valor de $0.933(>0,7)$ respecto la totalidad de la escala, resultando ser un valor muy satisfactorio.

En el AFE, se considera la rotación VARIMAX, ya que esta es la que proporciona la mayor interpretabilidad de los factores por dar lugar a una separación más clara entre ellos (Hair et al., 2009). Para calcular el número de factores a ser extraídos, se han utilizado los criterios de la raíz latente (autovalor correspondiente mayor a la unidad), del contraste de caída (patrón de tendencia en el diagrama de autovalores) y el análisis paralelo de Horn (1965) (comparación con los factores originados al azar por una matriz aleatoria de las mismas dimensiones). En base a estos métodos, se han seleccionado cuatro factores que explican en su conjunto el $53,7 \%$ de la varianza total de los datos. Para asumir la composición de los factores se ha considerado un valor mínimo de 0,5 como índice de contribución de los ítems. En la tabla 1 se presentan los resultados obtenidos: los factores detectados, los ítems que los conforman, el porcentaje de variabilidad explicada por el factor y el coeficiente de consistencia interna de cada uno de ellos. 
UN ESTUDIO COMPARADO SOBRE LA TRANSICIÓN DE SECUNDARIA HACIA LA UNIVERSIDAD ENTRE BRASIL Y ESPAÑA

Tabla 1. Composición factorial del instrumento

\begin{tabular}{llll}
\hline Dimensiones & $\begin{array}{l}\text { Varianza } \\
\text { explicada }\end{array}$ & $\begin{array}{l}\text { Alfa de } \\
\text { Cronbach }\end{array}$ & Ítems en cada factor \\
\hline F1: Adaptación académica & $33.2 \%$ & 0,934 & $\begin{array}{l}3,4,7,8,10,11,12,19,22,26,27, \\
32,33,34\end{array}$ \\
& & & $1,2,5,14,18$ \\
F2: Adaptación la nueva vida & $11 \%$ & 0,947 & $24,25,28,29,30$ \\
$\begin{array}{l}\text { F3: Adaptación a información, } \\
\text { horarios y recursos }\end{array}$ & $5,4 \%$ & 0,719 & $2,1 \%$ \\
F4: Autoestima y autonomía & $4,1 \%$ & 0,360 & 9,21 \\
\hline Total acumulado & $53,7 \%$ & 0,933 &
\end{tabular}

Fuente. Elaboración propia

\subsubsection{Análisis estadísticos}

Los niveles de los factores obtenidos mediante el AFE se analizarán en función del sexo (t-test); de la calificación en la prueba de acceso a la universidad (ANOVA); del lugar de residencia de participante (t-test); del nivel educativo de los padres (ANOVA) y del país de residencia del participante (t-test). Lo apropiado de los métodos paramétricos para comparar los niveles medios de los factores viene dada por la no significación de la prueba de normalidad de Shapiro-Wilk $(\mathrm{P}>0,10)$ y la robustez de los mismos ante el tamaño muestral que se considera en este trabajo (aplicación del Teorema del Límite Central). En los casos en que haya significación se indicará el tamaño del efecto estandarizado, d de Cohen en las comparaciones entre dos grupos y $\eta^{2}$ cuando el número de grupos a comparar sea mayor. Se suele admitir que $\mathrm{d} \approx 0,2$ indica un efecto de pequeña magnitud, $\mathrm{d} \approx 0,5$ es un efecto intermedio y $\mathrm{d} \geq 0,8$ indica un efecto importante; por su parte $\eta^{2}$ es la proporción de variabilidad observada en el factor que queda explicada por los niveles de la variable de agrupación. Para el tratamiento estadístico de los datos se considera el paquete IBM-SPSS 23.0. Se asume significación estadística siempre que el nivel de significación sea $P<0.05$.

\section{RESULTADOS}

Inicialmente se observa que la puntuación mínima en los ítems 1, 2, 5, 14 y 18 toman el valor cero $(0=$ no contesta) en lugar de 1 , como ocurre en los restantes ítems. Esto se debe a aquellos casos en que los participantes siguen teniendo el hogar familiar como lugar de residencia y por lo tanto ha elegido no contestar a estos ítems. Por otro lado, en 19 de los 35 ítems, el valor del tercer cuartil (3) refleja que hasta el 75\% de los participantes han contestado que no tienen una respuesta definida, o bien que están en desacuerdo.

El análisis de la distribución de las puntuaciones factoriales (diagramas de frecuencias y test de Shapiro-Wilk) permite asumir la distribución Normal para los factores 1, 3 y 4 sin embargo, el factor 3 presenta una distribución bimodal. Aunque no ha sido posible encontrar cuál es la causa de la bimodalidad. Como se ha indicado en la metodología, el suficiente tamaño muestral y la robustez de los t-test y de los métodos de tipo ANOVA 
permiten comparar las puntuaciones factoriales mediante métodos paramétricos en todos los casos. Por tanto, el análisis de los factores se ha llevado a cabo comparando los niveles del factor en función del sexo; de la calificación en la prueba de acceso a la universidad; del lugar de residencia de participante; del nivel educativo de los padres y del país de residencia del participante mediante test de Student o ANOVA en función del número de categorías de cada variable de agrupación.

El test de Student para muestras independientes no ha encontrado diferencias significativas entre los sexos en relación a los cuatro factores (en todos los casos $p>0,5$ ).

El ANOVA para la calificación de acceso a la Universidad no ha detectado diferencias significativas en función de la calificación obtenida en las pruebas de acceso (en todos los casos es $P>0,60$ ). Sin embargo, el estudio de Pérez-Ferra et al. (2018), que ha utilizado el mismo instrumento en población española, ha encontrado diferencias significativas entre esta variable y algunos ítems como la facilidad en comunicarse con los profesores, la utilización de la biblioteca o la adaptación a los horarios universitarios.

En el análisis de los factores relacionado con el domicilio del estudiante durante el curso académico, no ha encontrado significación estadística al comparar con primer factor $\mathrm{F} 1\left(\mathrm{t}_{(333)}=0,2 ; P=0,870>0,5\right)$, pero sí que se detectan diferencias entre los niveles de los factores restantes F2, F3 y F4. Por una parte, el alumnado que vive fuera del hogar familiar se adapta con mayor facilidad a la nueva vida (factor $\mathrm{F} 2$ : $\mathrm{t}_{(333)}=32,65 ; P<0,001$ ) y el tamaño del efecto estandarizado es considerable $d=3,58(0,8)$. Por otra parte, el alumnado que vive con la familia durante la carrera se adapta mejor a la información, horarios y recursos (factor $\mathrm{F} 3: \mathrm{t}_{(333)}=2,4 ; P=0,017$; sin embargo, se trata de un efecto de pequeña magnitud: $\mathrm{d}=0,27$ ), pero también presentan más dificultades de autoestima y autonomía (factor F4: $\mathrm{t}_{(333)}=2,2 ; P=0,026, \mathrm{~d}=0,24$ el efecto también es de pequeña magnitud). Estos resultados también son corroborados por el estudio, utilizando el mismo cuestionario, de Pérez-Ferra et al. (2018), que ha encontrado diferencias significativas cuanto al domicilio del estudiante durante el curso escolar.

En cuanto al Grado que cursa el alumnado participante, los resultados reflejan que aquellos que estudian Educación Primaria demuestran mejor adaptación a la vida académica universitaria que el alumnado de Pedagogía y Educación Infantil $\left(\mathrm{F}_{(2 ; 334)}=9,6 ; P<0,001\right.$, aunque solo se explica un $6 \%$ de la variabilidad del factor: $\left.\eta^{2}=0,06\right)$. Por otro lado, el alumnado de Pedagogía alcanza puntuaciones más altas en los demás factores, es decir, se adapta mejor a los cambios generados por irse a vivir fuera del hogar familiar $\left(\mathrm{F}_{(2 ; 334)}\right.$ $=8,5 ; P<0,001$; el tamaño del efecto también es pequeño $\left.\eta^{2}=0,05\right)$, se adapta mejor a la información, horarios y recursos $\left(\mathrm{F}_{(2 ; 334)}=67,4 ; P=0,001\right.$, el efecto ahora es bastante mayor: $\eta^{2}=0,29$, casi un $30 \%$ de variabilidad explicada), pero muestran menor grado de autonomía y autoestima $\left(\mathrm{F}_{(2 ; 334)}=69,4 ; P<0,001\right.$ el efecto también se puede considerar importante $\left.\eta^{2}=0,30\right)$.

\subsection{ANÁLISIS COMPARATIVO ENTRE PAÍSES}

La distribución por sexos en las titulaciones españolas es más homogénea que en Brasil. En España cerca del 23\% de los estudiantes de educación son hombres, frente al 76,8\% de mujeres, mientras en Brasil solamente un $2 \%$ de los estudiantes pertenece al sexo masculino.

Las edades de los participantes son semejantes en ambos países, pues el $80 \%$ se encuentra en la franja de los 17 a 21 años de edad. 
Respecto a la calificación obtenida en las pruebas de acceso a la universidad, se ha encontrado que cerca del $86 \%$ de los estudiantes brasileños han obtenido un Aprobado o Notable y solamente un 14\% ha aprobado con Sobresaliente. En España cerca del 77,5\% ha ingresado con un Aprobado o Notable, pero hay cerca del $22 \%$ que han sido calificados con un Sobresaliente. No obstante, como se ha dicho anteriormente, no se encontraron diferencias significativas.

El 72,0\% de los estudiantes brasileños viven con su familia durante el curso académico frente al 50,2\% de los españoles (test de comparación de dos proporciones independientes $P=0,004)$.

Con respecto al nivel educativo familiar se ha encontrado que en Brasil solo un 14\% de los familiares tienen estudios a nivel básico (Primaria y Secundaria), mientras el 76\% de los familiares tienen estudios universitarios y un $10 \%$ tiene el título de Doctor. En España, casi al revés de Brasil, la mayoría de las familias (68\%) tiene solamente los estudios básicos y cerca del $31 \%$ tuvo acceso a los estudios universitaria, mientras únicamente el 1,1\% ha alcanzado el título de Doctor.

Al comparar los niveles medios de los factores en función de los dos países participantes en el estudio, no se detectan diferencias significativas en el primer factor $\left(\mathrm{t}_{(81,13)}=1,28 ; P\right.$ $=0,205)$, lo que permite asumir que el alumnado de ambos países demuestra la misma capacidad para adaptarse a la vida académica en la universidad. No obstante, el alumnado brasileño alcanza puntuaciones significativamente más altas en los demás factores. Los resultados son: $\mathrm{t}_{(334)}=2,85 ; P=0,005$, con un efecto relevante $\mathrm{d}=0,44$ para $\mathrm{F} 2 ; \mathrm{t}_{(334)}=11,4$; $P<0,001$ con un efecto muy destacable $\mathrm{d}=1,75$ para F3 y $\mathrm{t}_{(56,1)}=7,8 ; P<0,001$ con un efecto también muy destacable d=1,64 para F4. Esto parece indicar que el alumnado de la Universidad brasileña afronta mejor el cambio hacia la nueva vida que aquellos que cursan la Universidad española, sin embargo, también tienen menos autoestima y autonomía.

\section{DISCUSIÓN Y CONCLUSIONES}

El domicilio del estudiante durante el curso escolar efectivamente tiene relación con su adaptación a la Universidad. Esta debería poder ofrecer servicios diferenciados de acogida, unos para aquellos que residen con sus familias mayormente centrados en el fortalecimiento de su autoestima y desarrollo de la autonomía, visto que vivir con la familia "inhibe" que los estudiantes asuman responsabilidades domésticas y de gestión económica. Por otro lado, la Universidad debería ofrecer, o mejorar, los servicios de orientación y psicopedagógicos con vistas a mejorar las competencias sociales y de relación interpersonal para el alumnado que reside lejos de la familia durante el curso.

Los resultados encontrados en este estudio ponen de manifiesto que los estudiantes de los Grados de Infantil (España) y de Pedagogía (Brasil) tienen más dificultades para adaptarse a la vida académica que el alumnado del Grado de Primaria. Este resultado, confirmado por el estudio de Pérez-Ferra et al. (2018), podría verse explicado por el mayor número de chicas que chicos en ambas titulaciones, que suelen tener desarrollar más arraigo en las relaciones de amistad que los hombres. Sin embargo, estudios anteriores apuntan hacia resultados opuestos, es decir, las mujeres tienen mejores resultados académicos en la universidad que los hombres (Rodríguez, Fita y Torrado, 2004), lo que se debe a mayor capacidad de esfuerzo, hábitos de estudio y de sobreponerse a situaciones adversas. En 
todo caso, indican la necesidad de mayor aporte preventivo por parte de la Universidad en proveer apoyos específicamente a los estudiantes de estas titulaciones, ampliando la oferta y la información sobre servicios de orientación que ayuden al colectivo con sus dificultades académicas (Connor, 2011).

Aunque no se hayan encontrado diferencias significativas en cuanto a la adaptación académica entre los dos países del estudio, se ha verificado que los estudiantes universitarios brasileños tienen más facilidad para ajustarse a la nueva situación social y cultural derivada del hecho de vivir lejos de la familia durante el curso académico, así como de adaptarse mejor a los aspectos institucionales, relacionados con el acceso a la información universitaria, los recursos ofrecidos y los horarios. Estos resultados podrían explicarse en función de una mayor capacidad de adaptación a la vida adulta por parte de los estudiantes de Brasil (Dutra-Tomé y Koller, 2014), o bien en función del mejor nivel socioeducativo de las familias de estos (OCDE, 2009). Si bien, como se argumentaba en la justificación ese proceso de adaptación a la vida académica no queda siempre justificado por el mayor nivel cultural y económico de las familias, como sucede en este caso con los estudiante de Brasil, ya que en las edades previas al ingreso en la Universidad se produce una mayor autonomía de los jóvenes, en relación a los padres, que hace que remita el seguimiento de las familias, con las consiguientes consecuencias para el aprendizaje y el posible desarrollo de la consolidación de la autonomía personal (Guevara et al., 2013; López-Betancourt et al., 2018). Pero si es cierto que los estudiantes más brillantes, en cuanto a calificaciones de acceso se comunicar con más fluidez con el profesorado; tienen más facilitad para acceder a las fuentes documentales y se adaptan más fácilmente a la nueva situación. No obstante, el alumnado brasileño, obtiene menores puntuaciones en lo que se refiere a la autonomía y autoestima, indicando la necesidad de que la Universidad refleje en la información y recursos brindados a los estudiantes de reciente ingreso, programas de apoyo personal y de orientación hacia el desarrollo de la autoestima.

Los resultados del estudio evidencian que es necesario desarrollar de modo más consistente la inteligencia emocional de los estudiantes; trabajar, tanto en la Universidad como en Secundaria, las inteligencias múltiples y orientar las acciones formativas según los puntos fuertes de los estudiantes en cada ámbito, del mismo modo, trabajar desde la perspectiva del Humanismo cívico en la disminución del fracaso de los estudiantes universitarios. Esa realidad se concreta en acogida, facilitando la información con una prosa clarificadora en los textos; realizando una tutorización entre iguales; mediante la asignación de un estudiante de cursos superiores que oriente a los estudiantes noveles; con la proximidad del profesorado que en no pocas ocasiones se le ve como inaccesible, entre otros aspectos.

\section{REFERENCIAS BIBLIOGRÁFICAS}

Almeida, L. S. y Soares, A. P. (2004). Os estudantes universitários: sucesso escolar e desenvolvimento psicossocial. En E. Mercuri y S.A.J. Polydoro (Org.), Estudante Universitário: características e experiências de formação (pp. 15-40). São Paulo: Cabral Editora e Livraria Universitária.

Andrade, C. Y. (2012). Acesso ao ensino superior no Brasil: equidade e desigualdade social. Ensino Superior, 6, 1-9. Recuperado de https://www.revistaensinosuperior.gr.unicamp.br/edicoes/ ed06_julho2012/Cibele_Yahn.pdf

Álvarez-Teruel, J. D., Tortosa-Ybañez, M. T. y Grau, S. (2012). La transición Secundaria - 
Estudios Pedagógicos XLVI, N 1: 79-92, 2020

UN ESTUDIO COMPARADO SOBRE LA TRANSICIÓN DE SECUNDARIA HACIA LA UNIVERSIDAD ENTRE BRASIL Y ESPAÑA

Universidad. Estrategia orientadora en la adolescencia. Revista de Psicología, 1(1), 557-566. Recuperado de http://www.redalyc.org/pdf/3498/349832342058.pdf

Bolsoni-Silva, A. T. y Loureiro, S. R. (2014). El papel de las habilidades sociales en la ansiedad social en estudiantes universitarios. Paidéia, 24(58), 223-232. doi: 10.1590/1982-43272458201410.

Brown, M. G., Wohn, D. Y. \& Ellison, N. (2016). Without a map: College access and the online practices of youth from lowincome communities. Computers y Education, 92, 104-116. doi: 10.1016/j.compedu.2015.10.001. recuperado de https://www.sciencedirect.com/science/article/ pii/S0360131515300506

Connor, D. J. (2011). Actively navigating the transition into college: narratives of students with learning disabilities. International Journal of Qualitative Studies in Education, 25(8), 10151036. doi: 10.1080/09518398.2011.590158. Recuperado de https://www.tandfonline.com/doi/ abs/10.1080/09518398.2011.590158

CSIC (s.f.). Webometrics Ranking of World Universities. Recuperado de http://www.webometrics. info/en/Latin_America/Brazil

Deci, E. L. \& Ryan, M. R. (1985). Intrinsic Motivation and Self-Determination in Human Behavior. Londres: Kluwer Academic.

Díaz-Barriga, A. (2013). Secuencias de aprendizaje. ¿Un problema del enfoque de competencias o un reencuentro con perspectivas didácticas? Profesorado. Revista de Currículum y Formación del Profesorado, 17(3), 11-13. Recuperado de http://www.redalyc.org/pdf/567/56729527002.pdf

Dutra-Thomé, L. y Koller, S.H. (2014). Emerging Adulthood in Brazilians of Differing Socioeconomic Status: Transition to Adulthood. Paidéia, 24(59), 313-322. Recuperado de https://pdfs. semanticscholar.org/9540/740149ed87444e4078871334befe15bd1c49.pdf

Fajardo Bullón, F., Maestre Campos, M., Felipe Castaño, E., León del Barco, B. y Polo del Río, M. I. (2017). Análisis del rendimiento académico de los alumnos de Educación Secundaria Obligatoria según las variables familiares. Educación XX1, 20(1), 209-232, doi: 10.5944/educXX1.17509. Recuperado de http://revistas.uned.es/index.php/educacionXX1/article/view/17509/18894

García Marco, F J. (1995). Fundamentos de organización y representación. Revista General de Información y Documentación, 5(2), 91-148. Recuperado de https://revistas.ucm.es/index.php/ RGID/article/viewFile/RGID9595220091A/11301

Gil Flores, J. (2011). Medición del nivel socioeconómico familiar en el alumnado de Educación Primaria. Revista de Educación, 362, 298-322. Recuperado de http://www.revistaeducacion. mec.es/doi/362_162.pdf

Guevara, E. P., Jaramillo, R. y Tovar, S. (2013). Factores familiares y su relación con el rendimiento académico en estudiantes de psicología. Revista Virtual Universidad Católica del Norte, 3(40), 122-140. Recuperado de http://www.redalyc.org/pdf/1942/194229200009.pdf

Hair, J. F., Black, W. C., Babin, B. J. \& Anderson, R. E. (2009). Multivariate Data Analysis. Harlow: Prentice Hall.

Horn, J. L. (1965). A rationale and test for the number of factors in factor analysis. Psychometrika, 30(2), 179-185. Recuperado de https://link.springer.com/article/10.1007/BF02289447

Jochen I., Menges, J. I., Tussing, D.V., Wihler, A. \& Grant, A. M. (2016). When Job Performance is All Relative: How Family Motivation Energizes Effort and Compensates for Intrinsic Motivation. Academy of Management Journal, 2(60), 76-91. Recuperado de https://journals. aom.org/doi/10.5465/amj.2014.0898

Jansen, E. P. W. A. \& Suhre, C. J. M. (2010). The effect of secondary school study skills preparation on first-year university achievement. Educational Studies, 36(5), 569-580. doi: https:// doi.org/10.1080/03055691003729070 . Recuperado de https://www.tandfonline.com/doi/ abs/10.1080/03055691003729070

Lawshe, C.H. (1975). A quantitative approach to content validity. Personnel Psychology, 28(4), 563575. Recuperado de http://citeseerx.ist.psu.edu/viewdoc/download?doi=10.1.1.460.9380\&rep= rep1\&type $=$ pdf 
López-Betancourt, A., García-Rodríguez, M. L. y Días-Soto, A. (2018). Hábitos de estudio y fracaso escolar en educación media superior. Praxis investigativa ReDIE: revista electrónica de la Red Durango de Investigadores Educativos, 10(19), 84-104. Recuperado de https://dialnet.unirioja. es/servlet/articulo?codigo $=6571560$

López-Cárdenas, I., Mella, J. y Cáceres, G. (2019). La universidad como ruptura en la trayectoria educativa: experiencias de transición de estudiantes egresados de Enseñanza Media Técnico Profesional que ingresan al Programa Académico de Bachillerato de la Universidad de Chile. Estudios pedagógicos, 44(3), 291-308. Recuperado de http://revistas.uach.cl/index.php/estped/ article/view/4233

Marini, J. A. da S. \& Boruchovitch, E. (2014). Self-Regulated Learning in Students of Pedagogy. Paidéia, 24(59), 323-330. doi: 10.1590/1982-43272459201406. Recuperado de http://www. scielo.br/scielo.php?script=sci_arttext\&pid=S0103-863X2014000300323

Martín-Mesa, A. y Alcalá-Olid, F. (2018). Observatorio económico de la provincia de Jaén, № 259. Jaén: Diputación provincial de Jaén/Universidad de Jaén. Recuperado de https://www.dipujaen. es/export/sites/default/galerias/galeriaDescargas/diputacion/dipujaen/observatorio_economico/ Numero259.pdf

McMillan, J. H. \& Schumacher, S. (2001). Research in education: A conceptual introduction. New York: Addison Wesley Longman.

Méndez-Giménez, A., Cecchini-Estrada, J. A. y Fernández-Río, J. (2017). Pasión, Motivación Autodeterminada y Autorregulación del Aprendizaje en el Deporte. Revista Iberoamericana de Diagnóstico y Evaluación - Avaliação Psicológica, 2(44), 146-157. https://doi.org/10.21865/ RIDEP44.2.12. Recuperado de https://www.aidep.org/sites/default/files/2017-09/R44-Art12.pdf

Ministério de Educação do Brasil (2017). Em 2014 58,5\% dos estudantes de 18 a 24 anos estavam na faculdade. Recuperado de http://www.brasil.gov.br/noticias/educacao-e-ciencia/2015/12/ numero-de-estudantes-universitarios-cresce-25-em-10-anos

Noels, K. A. (2001). New orientations in language learning motivation: Towards a model of intrinsic, extrinsic, and integrative orientations and motivation. In Z. Dornyei \& R. Schmidt (Eds.), Motivation and Second language acquisition (43 - 68). Honolulu: University of Hawaii Press.

Noels, K. A., Chaffee, K. E., Michalyk, M. \& Sugita McEown, M. (2014). Culture, autonomy and the self in language learning. In K. Cziser \& M. Magid (Eds.), The impact of self-concept on second language acquisition (pp.131-154). Bristol: Multilingual Matters.

Novo-Corti, I., Muñoz-Cantero, J. M. y Calvo-Porral, C. (2011). Análisis de las actitudes de los jóvenes universitarios hacia la discapacidad: un enfoque desde la teoría de la acción razonada. Relieve, 17(2), 1-26. Recuperado de https://www.uv.es/RELIEVE/v17n2/RELIEVE v17n25.pdf

Núñez, J.C. (2009). Motivación, Aprendizaje y Rendimiento Académico. Actas del X Congreso Internacional Galego-Portugués de Psicopedagogía. Braga: Universidade do Minho.

OCDE (2009). PISA 2009. Programa para la Evaluación Internacional de los Alumnos OCDE. Informe Español. Madrid: Ministerio de Educación. Recuperado de https://www.mecd.gob.es/ $\mathrm{dctm} /$ ievaluacion/internacional/pisa-2009-con-escudo.pdf?documentId=0901e72b808ee4fd

Pachane, G. G. (2004). A experiência universitária e sua contribuição ao desenvolvimento pessoal do aluno. En E. Mercuri y S. A. J. Polydoro (Org.), Estudante Universitário: características e experiências de formação (pp. 155-186). São Paulo: Cabral Editora e Livraria Universitária.

Pereira, E.M.A (2004). A percepção dos universitários sobre seus problemas. En E. Mercuri y S.A.J. Polydoro (Org.), Estudante Universitário: características e experiências de formação (pp. 187218). São Paulo: Cabral Editora e Livraria Universitária.

Pereira,A.yCardoso,F. (2015). Suicidal ideationin University Students: prevalence and Association with school and gender. Paidéia, 25(62), 299-306. doi:10.1590/1982-43272562201503. Recuperado de http://www.scielo.br/scielo.php?script=sci_arttext\&pid=S0103-863X2015000300299

Pérez-Ferra, M., Quijano-López, R. y Muñoz-Galiano, I. (2018). Transición de Secundaria a la Universidad en estudiantes de los títulos de maestro de educación Infantil y Primaria de la 
Universidad de Jaén. Aula Abierta, 47(2), 167-176. Recuperado de: https://dialnet.unirioja.es/ servlet/articulo?codigo $=6395876$

Raya Díez, E. (2017). Introducción a la metodología de aprendizaje servicio como metodología activa. En A.M. Vega Gutiérrez (Ed.), Los derechos humanos en la Educación superior: Enfoques pedagógicos innovadores a través del aprendizaje-servicio y del aprendizaje basado en competencias (pp. 131-152). Logroño: Comisión Europea.

Rio Grande do Sul tem a terceira maior renda per capita. (01 de marzo de 2018). Jornal do Comercio. Recuperado de https://www.jornaldocomercio.com/_conteudo/2018/02/economia/614023-riogrande-do-sul-tem-a-terceira-maior-renda-per-capita.html

Rodríguez, S., Fita, E. y Torrado, M. (2004). El rendimiento académico en la transición Secundaria-Universidad. Revista de Educación, 334(1), 391-414. Recuperado de http://www. revistaeducacion.educacion.es/re334/re334_22.pdf

Rodríguez Gallego, M. R. (2013). Metodología activa para la mejora del rendimiento en la educación superior. Revista Iberoamericana de Educación, 58(3), 1-9. Recuperado de https://rieoei.org/ RIE/article/view/1436

Rosario, P.S.L, Almeida, L.S., Guimarães, C., Faria, A., Prata, L., Dias, M. y Nuñez, C. (2000). As abordagens dos alunos à aprendizagem em função da área académica: Uma investigação na Universidade do Minho. En A.P. Soares, A. Osorio, J.V. Capela, L.S. Almeida, R.M. Vasconcelos y S.M. Caires (Eds), Transição para o ensino superior (pp.133-145). Braga: Conselho Académico, Universidade do Minho.

Runte-Geidel, A. \& Femia, P. (2015). Shadow Education in Spain: Examining Social Inequalities Through the Analysis of PISA Results. European Education, 47(2), 117-136, doi: 10.1080/10564934.2015.1033667. Recuperado de https://www.tandfonline.com/doi/abs/10.10 80/10564934.2015.1033667

Ryan, R. M. \& Deci, E. L. (2000). La teoría de la autodeterminación y la facilitación de la motivación intrínseca, el desarrollo social y el bienestar. American Psychologist, 55(1), 6878. doi: 10.1037110003-066X.55.1.68. Recuperado de http://www.davidtrotzig.com/uploads/ articulos/2000_ryandeci_spanishampsych.pdf

Texeira, M. A. P., Castro, R. D. y Piccolo, L. R. (2007). Adaptação à Universidade em estudantes universitários: um estudo correlacional. Interação em Psicologia, 11(2), 211-220.

Valadas, S. y Gonçalves, F. (2002). As abordagens à aprendizagem pelos estudantes da Universidade do Algarve. En S. Neves (Org.), Pedagogía e apoio psicológico no Ensino Superior (pp. 1- 22). Coimbra: Quarteto.

Vallerand, R. J. (2008). On the psychology of passion: in search of what makes people's lives most worth living. Canadian Psychology, 49(1), 1-13. Recuperado de https://selfdeterminationtheory. org/SDT/documents/2008_Vallerand_CanPsych.pdf 